Effect on scattering of complex morphology of DKDP bulk damage sites

C. W. Carr, M. D. Feit, J. J. Muyco, A. M. Rubenchik

November 10, 2004

Boulder Damage Symposium XXXVI Annual Symposium Boulder, CO, United States September 20, 2004 through September 22, 2004 
This document was prepared as an account of work sponsored by an agency of the United States Government. Neither the United States Government nor the University of California nor any of their employees, makes any warranty, express or implied, or assumes any legal liability or responsibility for the accuracy, completeness, or usefulness of any information, apparatus, product, or process disclosed, or represents that its use would not infringe privately owned rights. Reference herein to any specific commercial product, process, or service by trade name, trademark, manufacturer, or otherwise, does not necessarily constitute or imply its endorsement, recommendation, or favoring by the United States Government or the University of California. The views and opinions of authors expressed herein do not necessarily state or reflect those of the United States Government or the University of California, and shall not be used for advertising or product endorsement purposes. 


\title{
Effect on scattering of complex morphology of DKDP bulk damage sites
}

\author{
C.W. Carr, M. D. Feit ${ }^{*}$ J. J. Muyco, A. M. Rubenchik \\ Lawrence Livermore National Laboratory \\ 7000 East Avenue, L-491 \\ Livermore, CA 94550
}

\begin{abstract}
Bulk damage sites in frequency conversion crystals scatter and/or absorb laser light leading to interference and downstream intensification. We find that laser induced bulk damage sites in DKDP exhibit a "shell" of structurally and/or chemically modified material surrounding a central core as indicated by SEM and optical micrographs and micro Raman spectral maps. We hypothesize that the modified material has been shock wave densified and estimate the amount of densification and its effect on scattering. A simple model indicates that densification of several percent is likely and that the scattering cross section may be larger than the geometric area of the inner core by an order of magnitude.
\end{abstract}

Keywords: laser induced damage, scattering, KDP, DKDP, densification

\section{INTRODUCTION}

Unlike fused silica where the most common type of damage induced by ns scale laser pulses is on the surface, the most common form of laser damage in frequency conversion crystals (KDP/DKDP) consists of small ("pinpoint") bulk damage sites. These damage sites tend not to grow with repeated irradiation ${ }^{1}$, but there can be many such sites. An important issue for laser operation with crystals having bulk damage sites, is scattering from these sites that leads to an increase in downstream beam contrast that can enhance laser-induced damage on downstream optics. Thus, it is important to estimate how many such pinpoints can be tolerated for a given level of beam contrast.

There has been some inconsistency in reported sizes of pinpoints. Optical imaging using monochromatic (HeNe) illumination $^{2}$ may lead to a larger apparent size than imaging ${ }^{3}$ using white light. Our observations, reported here, indicate that DKDP bulk damage sites have a complicated morphology consisting of a "rubble-like" or perhaps empty core surrounded by a shell of modified material, which may be crystalline in nature. We hypothesize that this modified material is slightly densified due to passage of the shockwave accompanying the damage initiation. MicroRaman spectroscopy confirms that the shell surrounding the core of the damage site is different from undamaged DKDP. We suggest part of the discrepancy in reported pinpoint size results from differing illumination schemes is simply due to the averaging that occurs with incoherent illumination, i.e. that monochromatic illumination more readily detects scattering from the modified material.

We developed a simple model to estimate the effect on scattering of this shell of modified material. This model predicts the largest effect would be for densifications of about $5 \%$ in which case the scattering cross section could be an order of magnitude larger than would be expected on the basis of the core size alone. The relative sizes of core and modified shell suggest that densification is of this order.

\footnotetext{
* Correspondence: 925 422-4128, feit1@llnl.gov
} 


\section{MODIFIED MATERIAL SURROUNDS BULK DAMAGE SITE}

Tripler cut DKDP samples containing bulk damage, initiated by exposure to $3 \mathrm{~ns}$ pulses of $355 \mathrm{~nm}$ laser radiation, were prepared for analysis by "cleaving". Exposed bulk damage sites were imaged with SEM. Raman spectra in the vicinity of damage sites were obtained with a spatial resolution of about 1 micron. Typical SEM images are shown in Fig.(1). Besides a central core region, which appears to be empty or contain only rubble, a surrounding shell is evident in the images. The large fractures in some of the images are due to the sample preparation as indicated by the presence of wake hackle. The shell is just barely visible in optical images (Fig. 2) and had not been noticed until the electron micrographs were made. The faint optical visibility has been found on every optical image inspected. This visibility suggests the shell region may have a slightly different refractive index than the surrounding material.

Fig.(3) is an optical micrograph of a damage site for which Raman spectra were taken. Again, the large fractures are due to sample preparation and not due to laser damage. Raman spectra were obtained in the vicinity of the damage site. The spectra at two particular sites, denoted by black dots in Fig.(3), are shown in Fig.(4). The spectrum at the black dot away from the damage site, shown in Fig.(4a), is typical of DKDP ${ }^{4}$. Indeed, the degree of deuteration can be determined by examining the spectrum ${ }^{5}$. Note particularly the strong line near $900 \mathrm{~cm}^{-1}$, which is well known to be due to vibrations of the phosphate anion $\mathrm{H}_{2} \mathrm{PO}_{4}$ in DKDP. The spectrum at the black dot in the shell of modified material, shown in Fig.(4b), differs both in the amplitude of lines evident in Fig.(4a) and by the addition of several new narrow lines. Note particularly the strong new line at $716 \mathrm{~cm}^{-1}$.
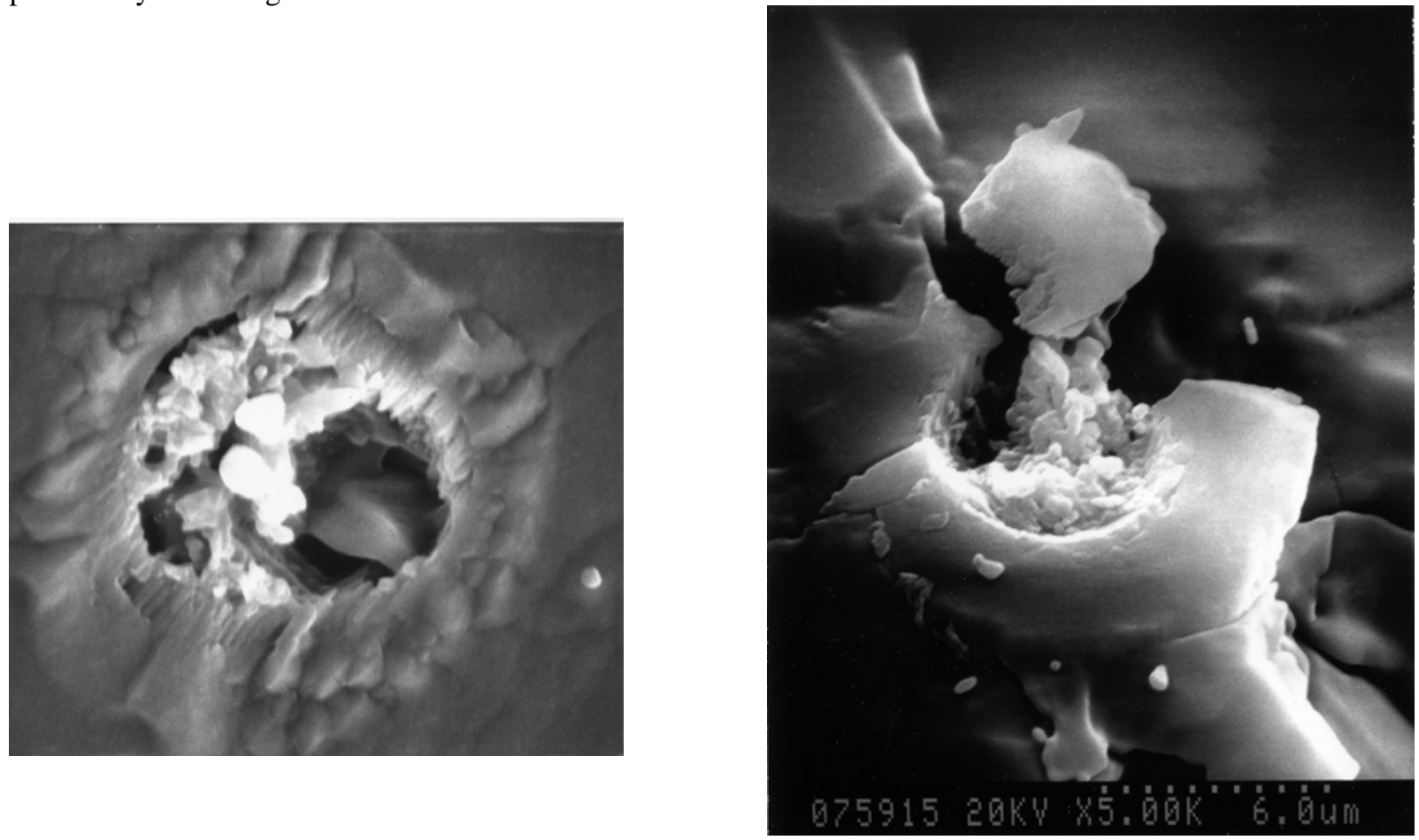

Fig. 1: SEM images of DKDP bulk damage sites initiated at $355 \mathrm{~nm}$. Sites are accessed by cleaving the damaged crystal. The large fractures seen in the right hand image are artifacts produced in breaking the crystal. 


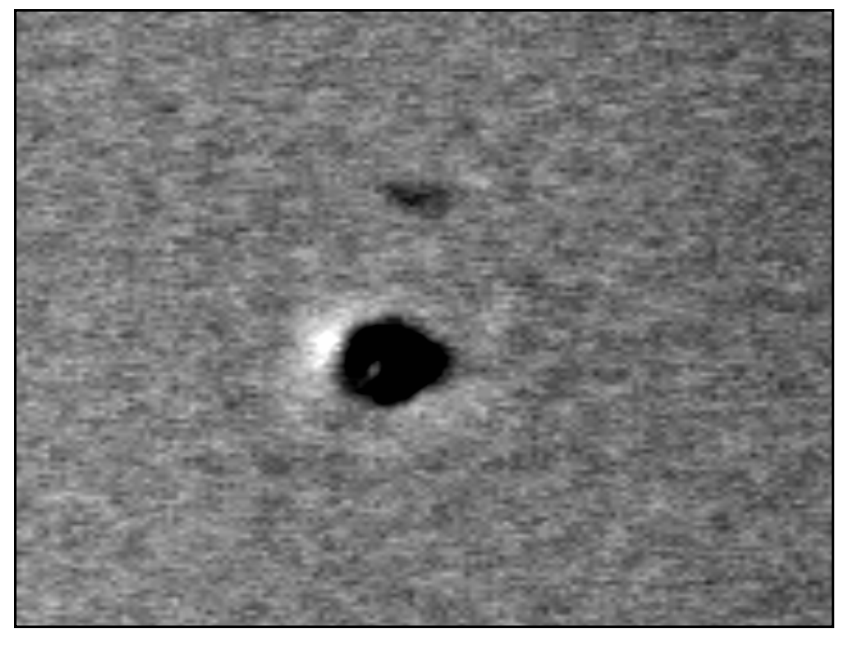

Fig. 2: Optical micrograph (with enhanced contrast) of bulk damage site showing evidence of modified region surrounding core.

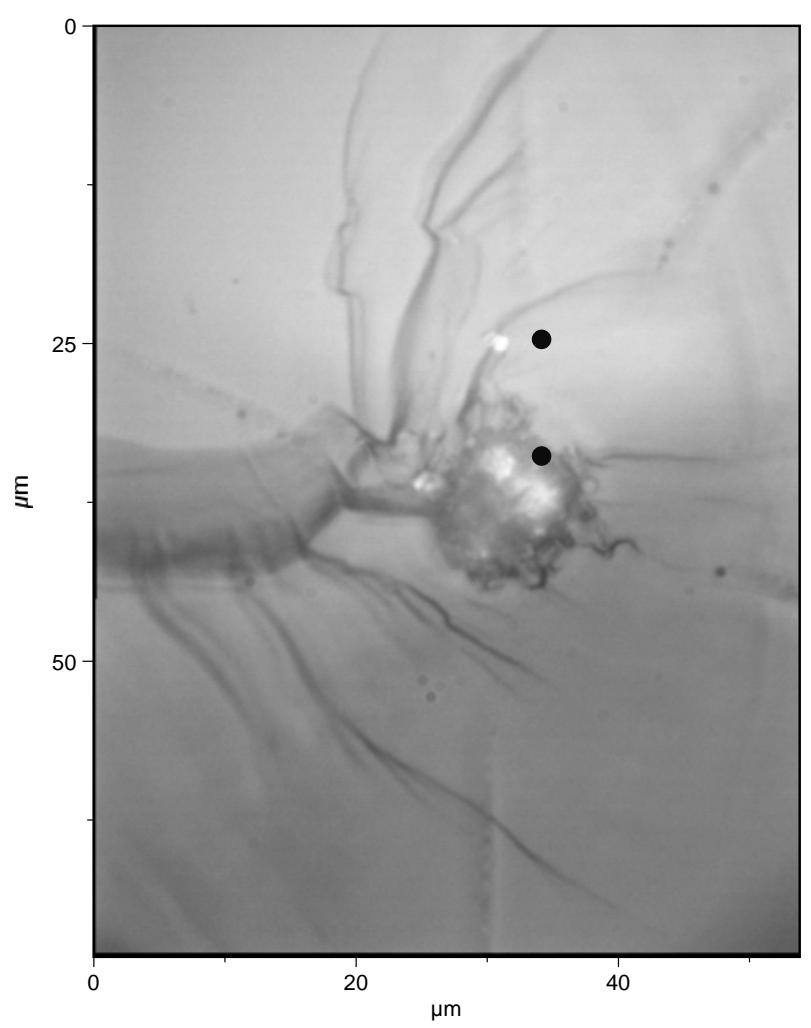

Fig. 3: Optical micrograph DKDP damage site. Large cracks due to cleaving crystal to gain access to bulk sites. Black dots indicate positions at which spectra of Fig.(4) were taken.

In addition to looking at the spectrum at a given location, we have mapped the intensity of spectral features in the vicinity of a damage site. For example, the distribution of the intensity at the $900 \mathrm{~cm}^{-1}$ frequency typical of DKDP shows a deficit in the core of the damage site. Alternatively, a map of the $716 \mathrm{~cm}^{-1}$ line found in Fig. (4b) exhibits the opposite distribution. Shown in Fig.(5), the $716 \mathrm{~cm}^{-1}$ line is found to have appreciable intensity only at the damage site, and especially in the shell region.

We have not unambiguously identified the origin of this new line. It is close to a series of Raman features found in a study $^{6}$ of stoichiometric changes in KDP accompanying surface damage caused by laser-induced breakdown. These features were attributed to $\mathrm{KPO}_{3}$ vibrations. However, in the present study, bulk sites are being studied and only a single narrow new line has been seen. The narrowness suggests a different crystalline phase is present. There may be a correlation with the pressure-induced change seen ${ }^{7}$ in room temperature DKDP hydrogen bonds. This possibility is presently being investigated.

Samples depicted in SEM images were prepared by first exposing to $10 \mathrm{~J} / \mathrm{cm}^{2} 3$-ns $355 \mathrm{~nm}$ laser radiation. A

$60 \mu \mathrm{m}$ x $3000 \mu \mathrm{m}$ beam was rastered over tens of square millimeters of sample after which the sample was scored and broken through the damaged region. The break was parallel or normal to the direction of laser propagation. The exposed surfaces were then evaporatively coated with $~ 200$ A of palladium to enhance electrical conductivity. The SEM images shown were taken with $10 \mathrm{KeV}$ electrons.

DKDP samples used for the micro Raman investigation were exposed to a small beam of $20 \mathrm{~J} / \mathrm{cm}^{2} 4-\mathrm{ns} 1065 \mathrm{~nm}$ radiation. The sample was then cleaved in the same manner as above. A micro-Raman instrument with a YAG doubled, diode pumped 36mW laser with a wavelength of $532 \mathrm{~nm}$ was used (Jobin Yvon Horiba HR800). Laser spot size on the 
sample was approximately $1 \mu \mathrm{m}$ in diameter using a 100x objective (Nikon CF Plan). The area map was obtained by moving the sample using a motorized XY stage with resolution of $0.1 \mu \mathrm{m}$ and reproducibility of $1 \mu \mathrm{m}$. The area covered was approximately $68 \mu \mathrm{m}$ in $\mathrm{X}$ by $47 \mu \mathrm{m}$ in $\mathrm{Y}$. Step size was $2.6 \mu \mathrm{m}$ in the $\mathrm{X}$ direction and $1.8 \mu \mathrm{m}$ in $\mathrm{Y}$. 27 points were sampled in each direction. Spectrum collection was for 90 seconds averaged over 3 collections at each point.

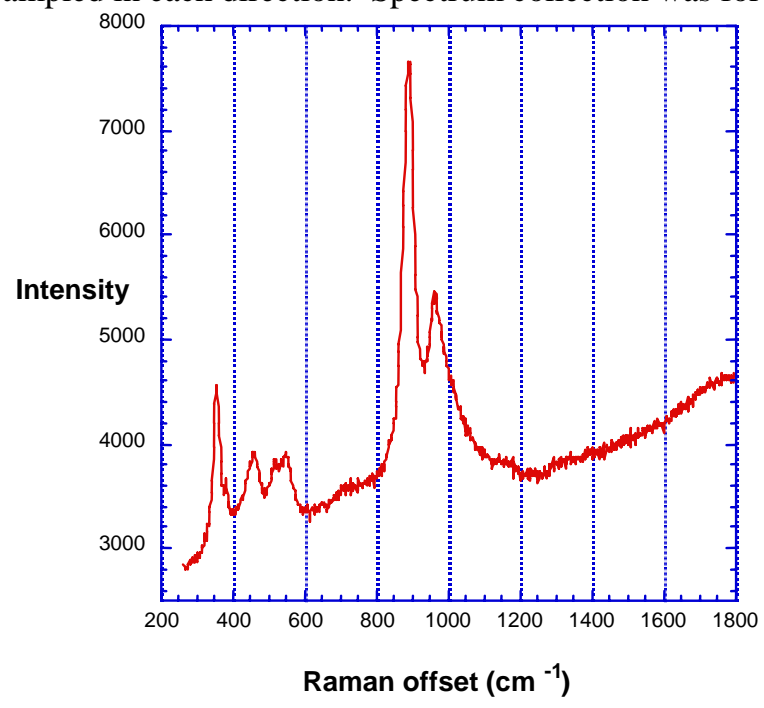

Fig. 4(a) Raman spectrum taken at a point away from the damage site.

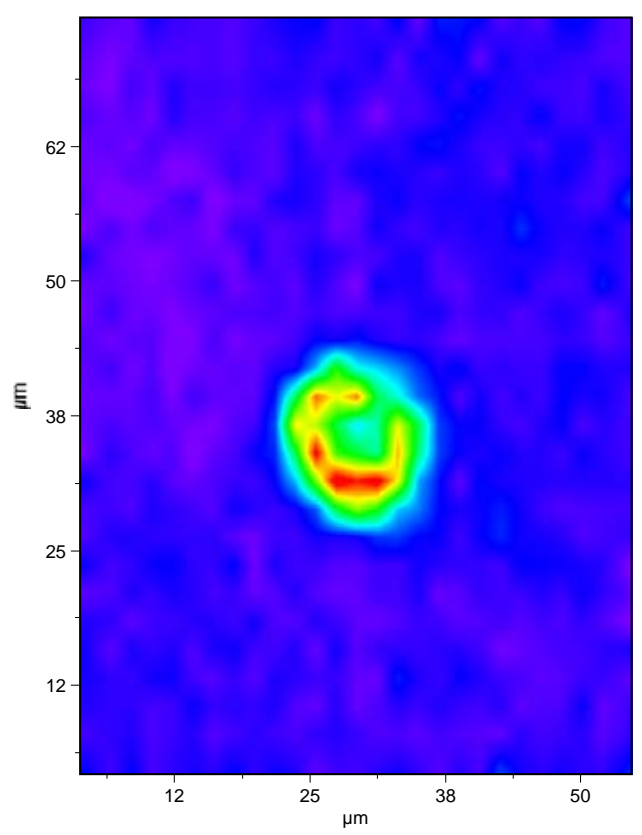

Fig. 5: Spatial map of the intensity of the $716 \mathrm{~cm}^{-1}$ Raman line near a damage site. This line only is evident at the damage site and is strongest in the shell around the core region.

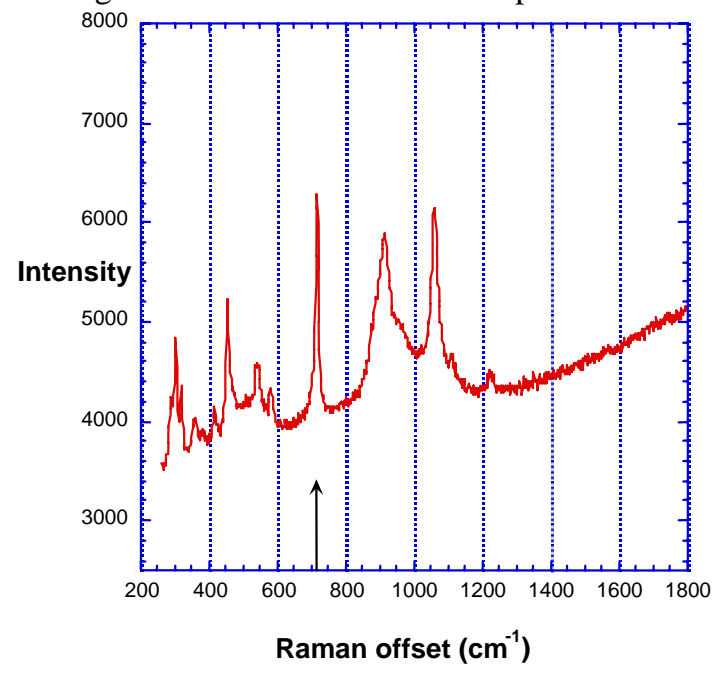

4(b) Raman spectrum taken at a point in the shell of modified material surrounding the core of a bulk damage site. Arrow denotes frequency corresponding to intensity map in Fig.(5). 


\section{MODEL FOR SCATTERING FROM DENSIFIED MATERIAL}

The experimental evidence suggests there is a shell of modified material surrounding the core of a bulk damage site in DKDP. This modified material probably has a slightly different refractive index from that of unmodified material. Assuming the material modification arises because of densification caused by the shock wave created during the damage event, we develop a simple model of densification and the importance of the shell region for scattering from a bulk damage site.

The idea is that damage initiation results in plasma formation (very hot vapor) within a small cavity of radius $\mathrm{a}_{0}$. After the laser pulse, the pressure in the vapor causes the cavity to expand to a final radius $\mathrm{a}_{\mathrm{m}}$. At the same time, the shockwave generated by the pressure in the cavity densifies material out to some outer radius $\mathrm{R}$ where the pressure has dropped below $\mathrm{P}^{*}$, the minimum pressure necessary to cause irreversible changes. The final results will depend on the values for $\mathrm{a}_{0}$, amount of deposited energy, and degree of densification that takes place. We will examine a reasonable range of parameters to delimit possible behaviors. We start from two simple principles:

$$
\begin{aligned}
& \boldsymbol{a}_{\boldsymbol{m}}=\boldsymbol{a}_{0}\left(\frac{\phi / \boldsymbol{a}_{0}}{2 \boldsymbol{P} *}\right)^{1 / 5} \\
& \boldsymbol{a}_{\boldsymbol{m}}{ }^{3}=\left(1-\frac{\rho_{0}}{\rho}\right) \boldsymbol{R}^{3}+\boldsymbol{a}_{0}^{3} \frac{\rho_{0}}{\rho}
\end{aligned}
$$

Here Eq.(1) describes adiabatic expansion of the central cavity assuming an ideal gas equation of state, $\mathrm{P}^{*}$ is the threshold pressure for irreversible change as described previously, and $\phi$ is the absorbed fluence. Eq.(2) just expresses conservation of mass. That is, the mass that originally was between radii $\mathrm{a}_{0}$ and $\mathrm{R}$ at initial density $\rho_{0}$, now is contained between radii $\mathrm{a}_{\mathrm{m}}$ and $\mathrm{R}$ at density $\rho$. For fixed assumed values of deposited laser energy and initial cavity size, we can use Eqs.(1) and (2) to calculate the final sizes of the core and modified shell regions as shown in Fig.(6).

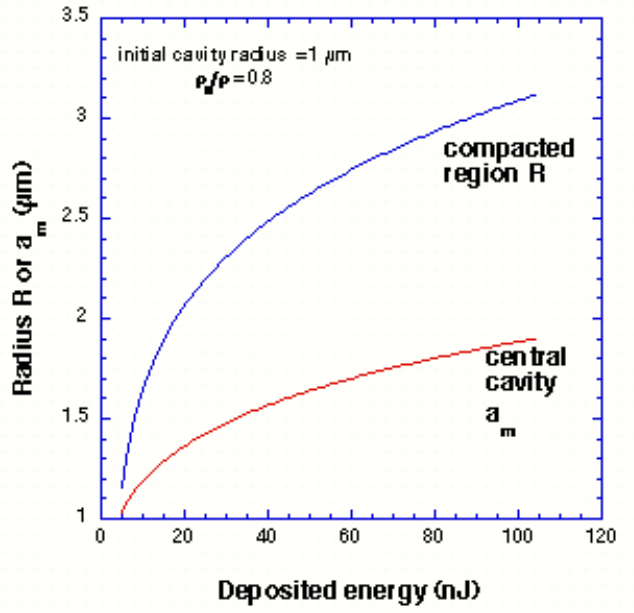

Fig. 6: Final radii of central core $\left(\mathrm{a}_{\mathrm{m}}\right)$ and compacted region $(\mathrm{R})$ as a function of deposited laser energy assuming densification of $20 \%\left(\rho_{0} / \rho=0.8\right)$ and initial core radius $\mathrm{a}_{0}$ of $1 \mu \mathrm{m}$.

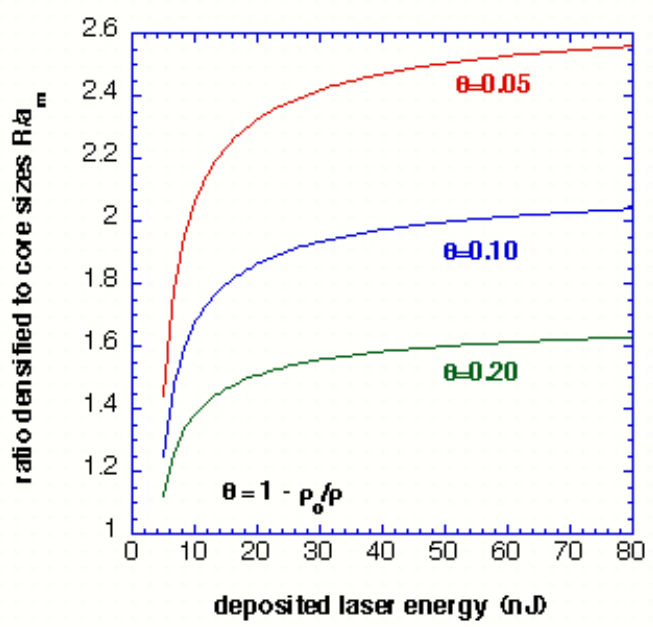

Fig. 7: Relative sizes of densified shell compared to core as a function of laser deposited energy and amount of densification.

It turns out that the relative sizes of the densified shell and inner core are not very sensitive to the deposited laser energy, but depend mostly on the degree of densification (Fig. (7)). Since the ratio of sizes of the two regions is known 
typically to be 2-4 (see Fig. (1)), this indicates that densification amounts to a few percent. The theoretical results reflect the fact that a smaller amount of densification can be caused over a larger volume by a given shock wave.

The effect on scattering can be estimated by making the reasonable assumption that the relative change in refractive index scales with density, i.e.

$$
\frac{\Delta \boldsymbol{n}}{\boldsymbol{n}} \approx \frac{\Delta \rho}{\rho}=\frac{\theta}{1-\theta} \approx \theta
$$

where $\theta=1-\rho_{0} / \rho$ as in Fig.(7). For moderate densification, the scattering cross section $\sigma$ can be calculated ${ }^{8}$ from

$$
\begin{gathered}
\sigma=\pi R^{2} G(2 k R \theta) \text {, where } k=\text { wavenumber and } \\
G(x)=2-4 \frac{\sin (x)}{x}+4 \frac{(1-\cos (x))}{x^{2}}
\end{gathered}
$$

Beside wavenumber, the argument $2 \mathrm{kR} \theta$ depends mostly on densification since

$$
2 \boldsymbol{k} \boldsymbol{R} \theta \approx 2 \boldsymbol{k} \boldsymbol{a}_{0}\left(\frac{\boldsymbol{P}_{0}}{\boldsymbol{P}_{*}}\right)^{1 / 5} \boldsymbol{\theta}^{2 / 3}
$$

There are two possible limiting cases. If the densification is very small, the change in refractive index vanishes and there is no additional scattering from the densified region. If the densification is large, then the extent of the densified region will be small and, again, there will be no additional scattering. This means there must be a maximum scattering cross section as a function of densification. This maximum, shown in Fig.(8), occurs in the densification range corresponding to the damage sizes observed experimentally.

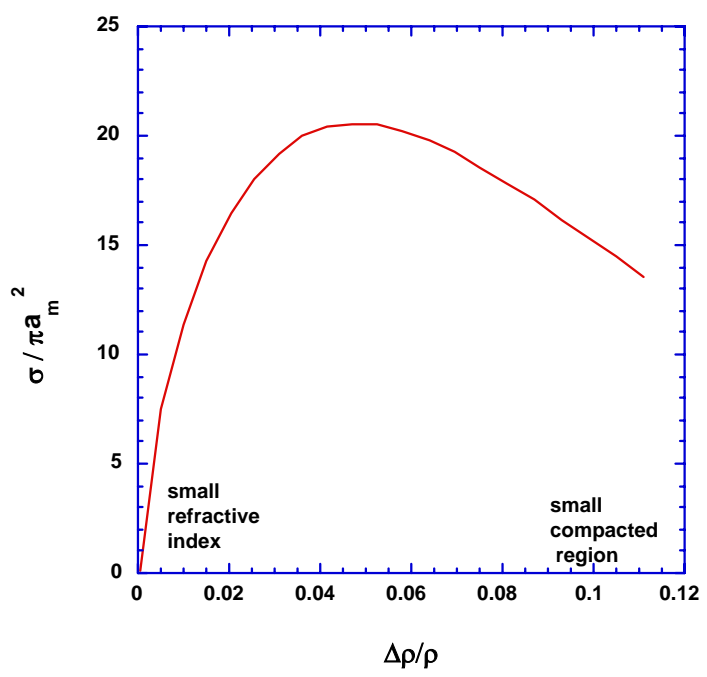

Fig. 8: Increase in scattering cross section due to densified shell surrounding core as a function of degree of densification. The largest effect is for densification of about $5 \%$ when the densified region is $2-3$ times larger than the core (see Fig. 7)

\section{APPARENT SIZE OF DAMAGE SITES}

Comparison of images taken in coherent vs. incoherent light (Fig.(9) show that apparent bulk damage sizes are larger in the former case. This is reasonable since scattering from small inhomogeneities tends to average out with white light illumination as can be verified using the scattering cross section of Eq.(4). Both the modified material of the shell and other inhomogeneities are revealed in coherent illumination. 

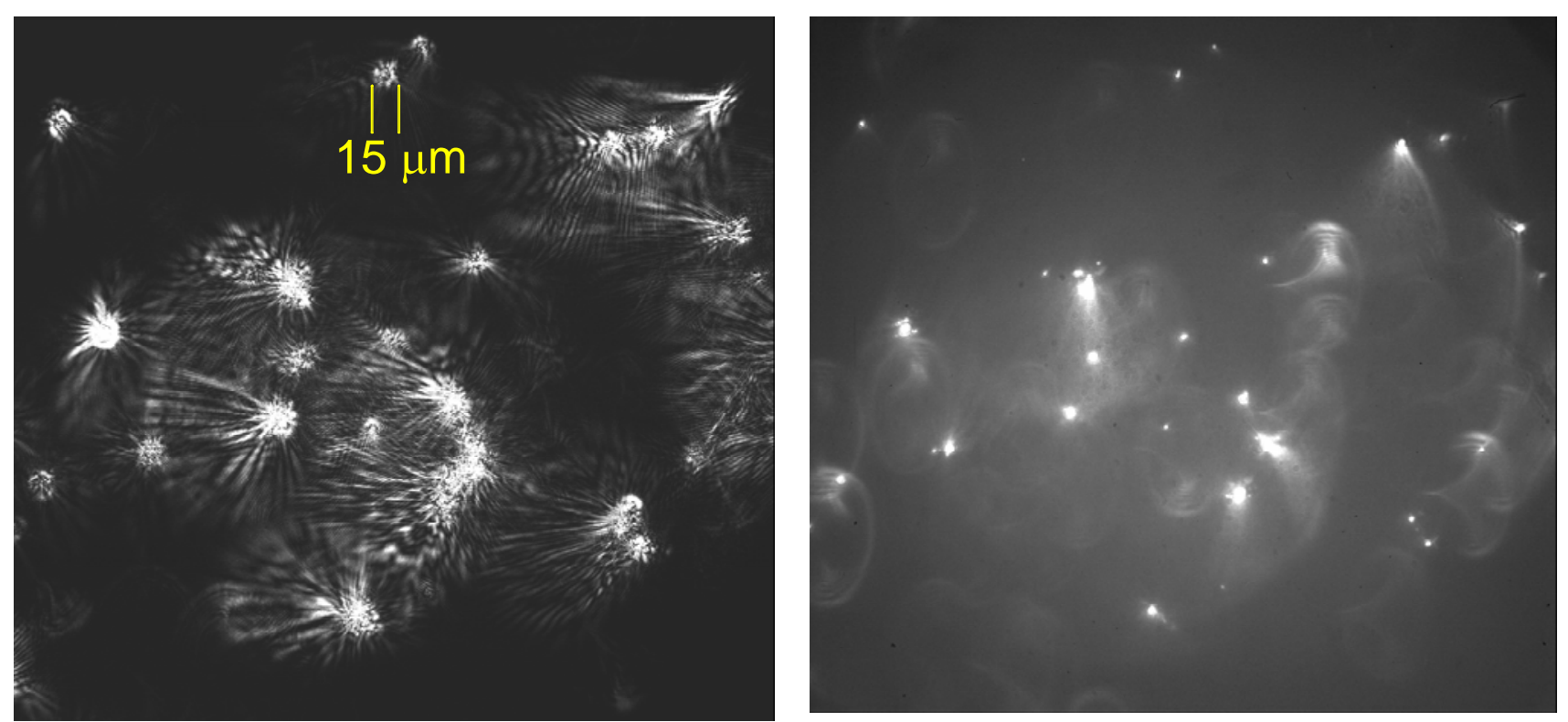

Fig. 9: Imaging of bulk damage sites with (left) HeNe beam and (right) with white light ${ }^{3}$.

\section{SUMMARY}

Laser induced bulk damage sites in DKDP exhibit a shell of modified material surrounding a central core or cavity. This modified material is visible in optical and electron micrographs and has a different Raman spectrum than undamaged DKDP. It is likely that this material is densified, perhaps crystalline, and has a slightly different refractive index than the undamaged material. A simple model allows the densification and effect on scattering to be estimated. This estimate predicts that scattering may be an order of magnitude larger than expected from the core size alone.

\section{ACKNOWLEDGMENTS}

This work was performed under the auspices of the U.S. Department of Energy by University of California Lawrence Livermore National Laboratory under contract No. W-7405-Eng-48

\section{REFERENCES}

${ }^{1}$ C.W. Carr, T.H. McMillan, M.C. Staggs, H.B. Radousky and S.G. Demos, "Evolution of bulk damage initiation in DKDP crystals”, Proc. SPIE 4932, 429-433 (2003)

${ }^{2}$ M. Runkel, A.K. Burnham, D. Milam, W. Sell, M.D. Feit and A.M. Rubenchik, "Results of pulse-scaling experiments on rapid growth DKDP triplers using the Optical Sciences Laser at 355 nm”, Proc. SPIE 4347, 359-370 (2001)

${ }^{3}$ P. DeMange, C. W. Carr, H. B. Radousky, and S. G. Demos "Microscopic characterization of laser-induced damage performance of large-size KDP and DKDP nonlinear crystals”, Proc. SPIE 5337, 47- 53 (2004)

${ }^{4}$ M.A. Yakshin, D.W. Kim, Y.S. Kim,Y.Y. Broslavets, O.E. Sidoryuk and S. Goldstein, "Determination of the degree of the deuteration degree of DKDP crystals by Raman spectroscopy technique, Laser Physics 7 (4), 941-945 (1997)

${ }^{5}$ This is implied in ref.[4] and shown explicitly by T. Huser, C.W. Hollars, W.J. Siekhaus, J.J. De Yoreo, T.I. Suratwala and T.A. Land, "Characterization of proton exchange layer profiles in KD2PO4 crystals by micro-Raman spectroscopy”, Appl. Spectr. 58, 349-355 (2004)

${ }^{6}$ R.A. Negres, S.O. Kucheyev, P.P. Demange, C.W. Carr, H.B. Radousky, S.G. Demos, "Stoichiometric changes to KH2PO4 during laser induced breakdown", this SPIE proceedings

${ }^{7}$ S. Endo, T. Chino, S. Tsuboi and K. Koto, "Pressure induced transition of the hydrogen bond in the ferroelectric compounds KH2PO4 and KD2PO4”,Nature 340, $452-455$ (10 Aug. 1989)

${ }^{8}$ H.C. van de Hulst, Light Scattering by Small Particles, Dover Publications, N.Y. (1981) 